\title{
The Effects of Lamp Spectral Distribution on Sky Glow over Observatories
}

\section{B. Luginbuhl ${ }^{1}$, P. A. Boley ${ }^{2}$, D. R. Davis ${ }^{3}$ and D. M. Duriscoe ${ }^{4}$}

${ }^{1}$ U.S. Naval Observatory Flagstaff Station, Flagstaff, Arizona, U.S.A. email: cbl@nofs.navy.mil ${ }^{2}$ Max-Planck-Institut für Astronomie, Heidelberg, Germany ${ }^{3}$ Planetary Science Institute, Tucson, Arizona, U.S.A. ${ }^{4}$ National Park Service, Bishop, California, U.S.A.

\begin{abstract}
Using a wavelength-generalized version of the Garstang (1991) model, we evaluate overhead sky glow as a function of distance up to $300 \mathrm{~km}$, from a variety of lamp types, including common gas discharge lamps and several types of LED lamps. We conclude for both professional, and especially cultural (visual), astronomy, that low-pressure sodium and narrowspectrum amber LED lamps cause much less sky glow than all broad-spectrum sources.
\end{abstract}

Keywords. atmospheric effects, scattering, site testing

We have modified the Garstang (1991) model to include wavelength-dependent scattering and absorption. We evaluate overhead $\left(\mathrm{z} \leqslant 60^{\circ}\right)$ sky glow at distances from $0.1-300 \mathrm{~km}$ from low-pressure sodium (LPS), amber LED (ALED; peak 590nm, FWHM 15nm), highpressure sodium (HPS), white LED with CCT of 2400K (wLED) and 5100K (cLED), metal halide with CCT of 4100K (MH), and a white LED with a 500nm filter (FLED). All lamp types are set to emit equal luminous flux. Results are summarized in Fig. 1.
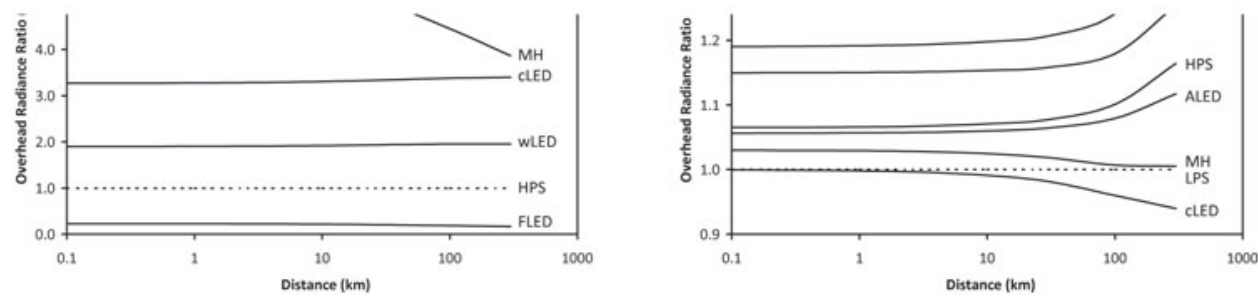

Figure 1. Ratio of overhead radiant sky glow as a function of distance. The left panel, relative to HPS, is for $\lambda 350-500 \mathrm{~nm}$ (LPS/ALED have no emission here); the right panel, relative to LPS, is for $\lambda 500-650 \mathrm{~nm}$. (Does not include natural sky glow)

In the range $\lambda 500-650 \mathrm{~nm}$, wLED and FLED cause $15-35 \%$ more overhead radiant sky glow (RSG) than LPS or ALED. Increased scattering at short wavelengths is balanced by increased extinction when observed from $<10 \mathrm{~km}$. At greater distances, RSG from MH and cLED decreases relative to LPS, while that from wLED, FLED and HPS increases. In the range $\lambda 350-500 \mathrm{~nm}$, wLED, cLED and $\mathrm{MH}$ contribute $\sim 2-5 \mathrm{x}$ more to the RSG than HPS. FLED has reduced blue RSG compared to broad-spectrum sources, but substantially greater blue and red RSG than LPS/ALED, especially at large distances.

Due to the Purkinje shift, narrow-spectrum yellow sources like LPS cause dramatically less visible sky glow $(1 / 2-1 / 9)$ than all broad-spectrum sources, including FLED.

\section{References}

Garstang, R. H. 1991, PASP, 103, 1109 